\title{
Designing a Web-based Decision Aid for Individuals to Consider Lung Cancer Screening
}

\author{
Pei-Yao Hung \\ School of Information \\ University of Michigan \\ Ann Arbor, MI, USA \\ peiyaoh@umich.edu \\ Mark S. Ackerman \\ School of Information and Department of EECS \\ University of Michigan \\ Ann Arbor, MI, USA \\ ackerm@umich.edu
}

\author{
Yan Kwan Lau \\ Department of Epidemiology \\ University of Michigan \\ Ann Arbor, MI, USA \\ yanlau@umich.edu \\ Rafael Meza \\ Department of Epidemiology \\ University of Michigan \\ Ann Arbor, MI, USA \\ rmeza@umich.edu
}

\begin{abstract}
Lung cancer screening, a relatively new procedure, is widely considered to be a promising way to address the low chances of survival from this disease by catching it at an earlier, more treatable stage. However, deciding whether to get this screening can be complicated for an individual; as such, patient decision aids are used to facilitate this process. To co-design a lung cancer decision aid, we conducted 5 participatory design workshops with 17 participants from African American and Latinx populations from communities with low resources. We also conducted 5 focus groups with 21 African-American participants to provide evaluative feedback. Through triangulating our fieldwork findings with other stakeholders, we detail the challenges of designing an inclusive decision aid and offer some suggestions with concrete examples, which complement current content-based guidelines, to assist in other patient decision aid development. We then discuss implications for applications to support decision-making in pervasive environments.
\end{abstract}

\section{CCS CONCEPTS}

- Human-centered computing $\rightarrow$ User centered design; Webbased interaction; - Applied computing $\rightarrow$ Life and medical sciences; Health informatics.

\section{KEYWORDS}

participatory design, patient decision support, cancer screening

\section{ACM Reference Format:}

Pei-Yao Hung, Yan Kwan Lau, Mark S. Ackerman, and Rafael Meza. 2019. Designing a Web-based Decision Aid for Individuals to Consider Lung Cancer Screening. In The 13th International Conference on Pervasive Computing Technologies for Healthcare (PervasiveHealth'19), May 20-23, 2019, Trento, Italy. ACM, New York, NY, USA, 10 pages. https://doi.org/10.1145/3329189.3329210

Permission to make digital or hard copies of part or all of this work for personal or classroom use is granted without fee provided that copies are not made or distributed for profit or commercial advantage and that copies bear this notice and the full citation on the first page. Copyrights for third-party components of this work must be honored For all other uses, contact the owner/author(s).

PervasiveHealth'19, May 20-23, 2019, Trento, Italy

(C) 2019 Copyright held by the owner/author(s)

ACM ISBN 978-1-4503-6126-2/19/05 ..\$15.00

https://doi.org/10.1145/3329189.3329210

\section{INTRODUCTION}

Cancer screening is an important public health intervention that involves the early detection of the disease when treatment is most effective. Unlike cervical, colorectal, prostate and breast cancer that have had population-wide screening for a few decades, lung cancer screening with low-dose computed tomography (CT) was only shown recently to be beneficial at reducing lung cancer mortality by $20 \%$ among those eligible [44]. Since lung cancer is the leading cause of cancer-related deaths in the U.S.A., with a larger burden than that of colorectal, prostate, and breast cancer deaths combined [37], being able to screen for lung cancer is an exciting development as it has the potential to improve the survival of this aggressive disease.

However, convincing people to think about lung cancer screening is challenging. Firstly, since it is a relatively new procedure, there is still a knowledge gap among both the targeted population and healthcare providers. In 2015, two years after it was recommended by the US Preventive Services Task Force (USPSTF), out of 6.8 million individuals who were eligible for the low-dose CT, only $3.9 \%$ were screened [15]. Secondly, it is also the first cancer screening modality recommended for the general population by national guidelines with eligibility criteria based not only on age (and sex), but also on a behavior - smoking history. Using smoking history as an eligibility criterion presents unique obstacles that other types of cancer screening do not have. For instance, an individual's smoking history may not be accurate due to the social nature of smoking, quit attempts, the stigma associated with being a smoker [42, 46], and the denialism of health risks and fatalism among smokers [40]. Moreover, individuals considered to be "low-risk" for lung cancer are not recommended to get screened while those who are at high risk (i.e., people who have smoked considerably and for a long time), are strongly recommended, since the benefits of screening would (most likely) outweigh the harms (i.e., anxiety, complications from follow-up tests such as a lung biopsy, and overdiagnosis). Other individuals may be eligible for screening, but their risk may be too low to benefit given the potential harms, and so the decision to screen becomes more of a complicated personal decision.

A decision aid is an evidence-based support tool that helps patients learn about a medical procedure/treatment, presenting the 
options and the potential benefits and harms, in the hopes that they can make an informed decision $[7,18]$. Lung screening guidelines by the USPSTF [26], as well as from Centers for Medicare and Medicaid Services (CMS) [16], recommend that patients use one or more decision aids to help them make an informed decision, and to facilitate a shared decision-making process with their healthcare providers. Increasing the relevance of decision aids, documenting the use of a decision aid is a requirement for healthcare providers to be reimbursed for the cost of the procedure for those covered by Medicare [16]. In response to this need, a web-based patient decision aid for lung cancer screening, shouldiscreen.com, was developed in 2014 [22], with a standard development process that cancer screening decision aids have traditionally employed [17]: i) literature review with grounding in the Ottawa Decision Support Framework; ii) expert review with clinicians and health risk communication scientists; iii) qualitative data from fieldwork with a working prototype; iv) pilot testing with an improved prototype; v) additional focus group feedback; vi) deployment. While this development was relatively successful, several aspects could be improved:

- The previous study was done in a predominantly white, and highly educated population in Ann Arbor, Michigan [21]. This is problematic as cancer disparities by race have been attributed to a lack of knowledge about cancer screening among minority populations [43, 47]. Moreover, African American men have the highest risk for lung cancer when compared to other demographic groups [28]. Not catering to groups that are at high risk and already have less access to care could further exacerbate existing disparities.

- Whilst this web-based decision aid satisfies the International Patient Decision Aid Standards instrument (IPDASi) criteria [18], a set of guidelines recommended for developers of patient decision aids used in the field of medicine, these criteria are not specific to the format/medium of the decision aid. Health communication experts were consulted in the development to ensure that the content was represented appropriately (i.e., risks, graphs, vocabulary), but no specific considerations were given to design features catering to the target audience, and preparing them for possible patientphysician interactions.

To address these limitations and explicitly embrace principles of human-centered design, we engaged current and former smokers in minority populations - African Americans and Latinx - in Metro Detroit, Michigan, USA, who enrolled in participatory design workshops. We also created a modified version of shouldiscreen.com which incorporated additional content based on the feedback from the participatory design workshops, for other community members to evaluate in focus groups. Through the results of this study, our paper offers the following contributions:

- Provide insights into a layperson's views on the design and content of a lung cancer screening decision aid through participatory design and evaluative focus groups.

- Discover challenges of designing a decision aid by comparing perceptions of lung cancer screening and the associated harms and benefits among laypeople and physicians.
- Identify three themes of design suggestions for web-based decision aids: vocabulary, time, and delivery, based on our findings above, to complement the existing IPDASi guidelines with specific consideration of design features.

\section{RELATED WORK}

\subsection{Cancer screening patient decision aids}

Decision aids have been used in the context of cancer screening for various purposes, when there is more than one reasonable option to choose from: i) to help decide between differing recommendations by medical groups (e.g., earlier starting age for mammograms for some moderate risk individuals), and ii) to help choose between different modalities (e.g., colonoscopy, sigmoidoscopy, fecal occult blood test, virtual colonoscopy, and DNA stool test, for colorectal cancer screening) each with different test characteristics and comfort levels [17]. For lung cancer screening, the decision is to get annual screening (single modality with low-dose CAT scan), or not to get screened, based on age and smoking history [26]. In public health research, the outcomes of interest for decision aids are clinical in nature (e.g., was the use of a decision aid associated with screening rates?) and their intermediaries such as knowledge, decisional conflict, perceived risk, etc., derived from health behavior theories such as the Transtheoretical Model [32], Health Belief Model [14], and the Ottawa Decision Support Framework [17, 29]. In the same vein, the IPDAS guidelines [7, 18], which purports to assess the quality of patient decision aids, focus mainly on the content of a decision aid. These criteria and outcomes are, to some extent, medium neutral. However, with the growing usage of the web to look for health information [10], more directed focus on the design on web-based resources is becoming increasingly important.

\subsection{Lung cancer screening decision aids}

Unlike other types of cancer screening, screening for lung cancer was the first of its kind where CMS required that shared decisionmaking take place in order for healthcare providers to be reimbursed by Medicare, a federally administered national health insurance program for individuals above 65 years old. The decision memo by CMS stated that shared decision-making for lung cancer screening must include [16]:

"the use of one or more decision aids, to include benefits and harms of screening, follow-up diagnostic testing, over-diagnosis, false positive rate, and total radiation exposure."

While this requirement specifies the use of decision aids, it does not specify how a decision aid should be used, or what medium it could be in (e.g., paper vs. web). Due to the nascency of this kind of requirement, there are not many decision aids that explicitly set out to fulfill IPDAS or CMS criteria, or that have been tested in users who may benefit from the decision aid. Below, we review and compare three decision aids for lung cancer screening that have been used/tested, and also fulfill these criteria, as it may be instructive to examine how design considerations were taken into account.

2.2.1 Lung cancer screening tools by Agency for Healthcare Research and Quality (AHRQ) [9]. 
AHRQ, an agency within the Department of Health and Human Services, developed a decision aid for lung cancer screening in the format of a one-page long website, with the option of printing out all the content on that page. Content wise, it fulfills the IPDAS criteria and CMS requirements and contains a values clarification exercise and a set of questions that a potential patient might have for when they see their doctors. The representation of the potential benefit from getting screened (i.e., relative mortality reduction) were two panels of icon arrays that display the number of deaths over 1000. The content is also available in Spanish. In terms of ease of navigation, having all the content on one page removes the confusion for the user with regards to "where to go next." However, having all the content on one page for individuals to scroll through and digest (equivalent to about 4 letter-sized pages) may be too overwhelming to absorb.

\subsubsection{CHOICE by University of North Carolina, USA [33].}

"Choice" is a video decision aid that is just over 6 minutes long (6:19) and satisfies the IPDAS and CMS criteria. The video is in the format of slides that are narrated, with content very similar to that of AHRQ's, but with more emphasis on getting people to quit smoking. A disadvantage to using video as a medium is that it may take more effort than web-based decision aids to update the content, which is likely necessary for lung cancer screening as more data are collected as screening coverage increases, and guidelines are updated.

\subsection{3 shouldiscreen.com by University of Michigan, USA [22].}

This is a web-based decision aid, one of the earliest ones developed in time for healthcare providers to use for the purposes of reimbursement from CMS. Same as the above two, this decision aid fulfills the IPDASi and CMS criteria. Unlike them, however, shouldiscreen.com also provides a way for individuals to calculate their 6-year risk/probability for developing lung cancer. This risk may be more clinically relevant as there appears to be a wide range of risks, even among screen-eligible individuals [1]. For those considered to be at low risk (below 1.5\%), the benefit of getting screened may not be as great as someone who is at high risk. There is no fixed navigation structure on the website which could be confusing for people who have not heard about lung cancer screening. Finally, there is a venue for users to provide feedback about the website.

\subsubsection{Other remarks.}

There are clear advantages of using a website over video for decision aids:

- The ability to update content/information readily is particularly critical in light of recent revisions to other cancer screening recommendations such as for cervical and prostrate due to the evidence base being updated. For lung cancer, some of the information about false positives from the test is already being contested as the diagnostic criteria have changed from the randomized controlled trial.

- Tailoring information relevant to an individual based on input. This is important because of the difference in insurance coverage by age range (Medicare vs. Non-Medicare), and also to frame the message differently for smokers vs. former smokers. Additionally, being able to estimate personalized risk is important for lung cancer screening specifically because there exists a large range of risk-benefit ratio from screening even for the screen-eligible population [1].

While decision aids have largely been viewed as tools patients can use in preparation to see their doctor and/or together with their healthcare provider to facilitate shared decision-making, the reality in the context of lung cancer screening is this: shared decisionmaking is probably not happening, at least not to an adequate enough degree, with the length of a typical discussion to be under a minute long [3]. We should also think about expanding the role of a decision aid to go beyond that one point in time at the doctor's office, to one where the patient learns about lung cancer screening as the process evolves. A decision aid can be a resource for them to refer back to for reassurance and/or be mentally prepared for what is to come between going to the primary care doctor to the radiologist, and between getting the $\mathrm{CT}$ scan and getting the results.

\subsection{UX of online health tools \& resources for older adults}

The Internet has become an important source of health information for adults above 45 years old, closely rivaling that of healthcare providers themselves: the latest cycle of the Health Information National Trends Survey in 2017 (HINTS) conducted by the National Cancer Institute showed that $45 \%$ looked for health information on the Internet; and when there was a strong need to get information about health/medical topics, $54 \%$ responded that they would first go see "doctors or healthcare providers" while $34 \%$ said that Internet would be their first port of call [12]. There has been a steady increase in efforts to understand the unique needs of older adults in their interactions with online/e-Health resources: more generally [49], care navigation [2], cancer navigation [11,13], and cancer education $[25,27]$. However, to the best of our knowledge, there has been little focus in the Human-Computer Interaction (HCI) arena with regards to patient decision support tools, which are challenging to design for as each target population of the health decision in question has special needs (e.g., women for mammography vs. men for prostate cancer screening). Our contribution, then, provides a flavor of design challenges and opportunities through our work to design a patient decision aid for lung cancer screening.

\section{METHODS}

With the goal of understanding laypeople's perspectives on how a decision aid can better engage them to think about lung cancer screening and understand the related harms and benefits, the research team hosted participatory design (PD) workshops [36] with current and former smokers from low-resource communities. We recruited African-American participants through a non-profit organization and Latinx participants through a local health clinic in Detroit. Five PD workshops took place, with a total of 17 participants, aged between 45 and 77. Among these participants, 13 are former smokers and 4 are current smokers.

The workshop consisted of four steps that were designed to explore laypeople's views on what they considered important with regards to lung cancer screening and also to obtain feedback about the content of an existing web-based decision aid [22]. First, participants were given a scenario that illustrated a situation where the 
main character was worried about a family member or loved one having lung cancer, and wondered about whether/how they could approach them about lung cancer screening. Participants were encouraged to pick their own family member or loved ones instead should they feel comfortable. Participants were then asked to write down any questions they might have regarding lung cancer screening. Second, participants teamed up in groups of two or three to collaboratively design a website or online resource that can answer the questions they had generated. Participants were instructed to sketch and draw on flip charts and use sticky notes to add any form of content if desired. Each group would show their design to the rest of the workshop participants to help everyone understand their design and get feedback. Third, after designing their own website, participants read a set of paper cut-outs that contained all the content from shouldiscreen.com, an existing web-based decision aid, and give feedback regarding 1) whether they could understand (and if not, identify the parts that were difficult), and 2) whether they found the information useful. Fourth, participants were asked to add these paper strips to where they felt suitable on the website they had designed on the flip charts. Throughout this process, a research assistant was assigned to each group to facilitate the discussion. The assistant also observed the design process and wrote down notes. We chose a group-based PD because lung cancer screening is a new, relatively unknown procedure. $\mathrm{PD}$ allows participants to engage in the design process by raising questions and discussing suggestions. Moreover, due to the stigma surrounding smokers, participants (i.e., current/former smokers) feel more comfortable expressing opinions to group members with similar experiences. Also, by having participants sketch their designs as the main PD activity, participants get a tangible artifact that visually documents their ideas and their importance, and the process of refining the design over time.

With regards to the analysis, there was a debrief among research assistants and the second author directly after the workshops leading to a brief report. The workshops were audio recorded and transcribed. Spanish transcripts were translated into English. We used thematic analysis to analyze the transcripts, notes, and participants' sketches to identify the major themes that provide insights into the design of a lung cancer screening decision aid that could be useful to an individual with the decision-making process. The first and second author coded and analyzed the sketches independently. The first author analyzed the transcripts of the workshops and discussed the findings with the second author to harmonize the themes gleaned directly after the workshops. We then created a version of shouldiscreen.com to include additional information regarding insurance coverage criteria, as we found it to be important for participants, and asked 21 individuals over 5 focus groups from the African-American community we had been working with who did not participate in the designing process to evaluate this version. Inclusion criteria for focus groups are the same for the PD workshops. Focus groups were used for triangulation as we felt the need to verify the workshop findings with focus group findings before major feature updates. The analytic procedure for the focus groups was similar, except that the first and second author independently coded the transcripts.

After we consolidated some preliminary findings and derived design implications from this round of design and evaluation, we presented this work to two community partners who provided access to their communities and to two physicians who specialize in lung cancer screening and have been working with patients in two health systems in Michigan, who also serve as the medical consultants for shouldiscreen.com. We conducted semi-structured interviews with the physicians and presented the results of the workshop and paused for reactions at each finding. We believe that even for a patient-centered tool, feedback from physicians would be important. Given that lung cancer screening is a relatively new procedure, patients may have misconceptions of risks and benefits that doctors can address. Moreover, patient-centered tools surrounding shared decision-making of medical procedures should explicitly support patient-doctor interaction - which we believe to be an important contribution to the HCI community. In the following section, we present findings and design suggestions with examples of possible features to demonstrate how the suggestions can guide developers of cancer screening decision aids in refining their design to better engage the audience.

\section{FINDINGS}

Through the participatory design workshops, focus groups, and feedback from physicians and our community partners, we identified three aspects - vocabulary, time, and delivery - where the differences between patients and physicians have made the current design inefficient. Below, we describe these through the perspectives of (potential) patients and physicians to demonstrate the challenges of designing decision aid. In the remainder of the paper, we will use "patient" to refer to people who might benefit from lung cancer screening.

\subsection{Vocabulary: We speak different languages, but we need to understand each other}

4.1.1 Patients. Patients were motivated to understand what was at stake, but needed a more familiar tone and language that speak to them. Since lung cancer screening is still largely unknown to the general public, patients need help to understand the relevance of lung cancer screening to them. For instance, one common factor brought up by patients was to talk about symptoms to which people might relate to stimulate the consideration of having lung cancer screening. Another suggestion that emerged was the use of regular folk who are not physicians who had gone through the screening to "speak to us," so as to better relate to their message. Patients also did not understand some of the medical vocabulary and explanations used on the existing web-based decision aid. Explanations for "nodules" and "CT (computed tomography) scan" were commonly requested. Another major barrier was the presentation of risk (a required item from IPDASi guidelines [18]) and its meaning. Risk presented in terms of probability and icon arrays were too abstract; instead, patients asked how their estimated risk would relate to potential outcomes of the screening, and the subsequent impact on their quality of life.

"What I'd like to see first is, not professionals, but average people talking... I want it to open up with people talking in layman's terms, and the conversation they have is who is at risk about lung cancer and things they see, what was [sic] the signs that they have, and 
then tell me there is a test to detect lung cancer." - Female smoker, participatory design workshop (African American)

Even when patients had no trouble reading and understanding the content, they might not 1) understand the rationale or 2) be able to extract the message that the decision aid developers hope to deliver, so end up being confused. During the workshops, when participants were asked to go through the content from the decision aid, they often ask these two types of questions 1) "I see... but why?" and 2) "Ok... so what?" These suggest that some of the content is either too concise or being too implicit about the underlying messages.

"I think it [lung cancer screening] should be before the age of 55. Why is it at the age of 55?"- Female smoker, participatory design workshop (African American)

To summarize, we found that while certain representations of information - bullet points, icon arrays, and tables - present information in a concise format, patients might find it too brief and still need help interpreting the available information.

4.1.2 Physicians. The physicians in general agreed with the need for an online decision aid to connect to the target audience better, possibly through more familiar tone and language. However, they also strongly believed that the design should not bias patients towards/against screening. For instance, in reaction to patients' suggestions of showing the relevance using familiar language and symptoms, as Doctor B stated,

"This is a difficult issue, and I do understand that people need to feel that it's important. On the other hand, part of this is a decision tool, and it's meant to be relatively neutral or balanced about things. And so, having a landing page that has too much of a hook can be an issue... How do you get people to know about the symptoms? The screening approach of old is they'll have something very scary sounding. Q: What are the signs that you have lung cancer? A: You'll feel fine. We are trying to move away from that."

The physicians, while understanding why the participants brought this up and had also received similar reactions from their patients, repeatedly emphasized that this would mislead people into thinking that symptoms are needed before people get screened.

Regarding the issue of medical vocabulary and interpretation of risk, the physicians acknowledged that while the decision aid might be informative for a patient if a health professional is present to help explain, the content is likely to be difficult for laypeople to digest. For instance, Doctor A mentioned his experience of having to explain the concept of nodules frequently to his patients:

"Phrases like 'nodules are like freckles'... I mean, they're like freckles, but not really... Freckles are on the skin, but they're very similar in that they're an 'imperfection' and it doesn't 'belong' but they're functionally inconsequential, right? And it's a disarming term, right? You can see the change in the patient's face, the tension, literally just drain away when you use terms like that."

\subsection{Time: My concept of what matters when is different to yours}

4.2.1 Patients. We observed that patients think about screening on different time scales. When given the scenario in the workshop and asked to think about questions they might have regarding lung cancer screening, the questions often followed a process or sequence of events (see Figure 1). These questions can generally be categorized into the following types: why should I care, what does the procedure involve, what are the side effects if I get screened, and what happens after getting the results if they are positive/negative. Naturally, different patients think about the timeline with different granularity. While some focused on the process of making the decision to screen and some focused on the screening process itself, others also recognized the possibility of follow-up procedures and treatment and asked more questions about the entire process.

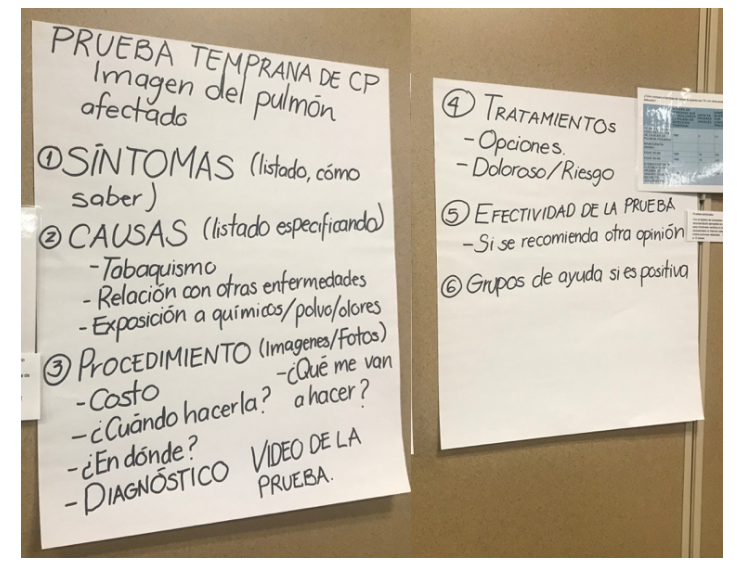

Figure 1: Landing page designed by a Spanish-speaking group that highlighted the themes in this order: 1) Symptoms; 2) Causes; 3) Procedure; 4) Treatment; 5) Effectiveness of the test; 6) Support groups if tested positive

4.2.2 Physicians. The physicians we interviewed reacted positively to the idea of screening being a process, but was surprised that some patients also held the same view. From a physician's experience, patients often assume that lung cancer screening is a one-time event and could result in good or bad news. For instance, Doctor A was surprised that our workshop participants asked questions about screening in a process-oriented way.

"You know, it's interesting to hear you use the terms concepts and flow. My perception is that patients think of lung cancer screening as a one-time event. I passed the test. I try to emphasize to patients that lung cancer screening is a process that takes place over multiple years, and I'm surprised that you hear that people describe it that way."

Of course, we should acknowledge that our participants might be different from people who have reached the stage where they are discuss with physicians regarding the possibility of going through lung cancer screening. Nevertheless, our observation from the workshops, focus groups, and interviews with physicians suggest the 
following two findings. First, potential patients do think about the screening process on different time scales. Second, potential patients' sense of time regarding lung cancer screening is different from what physicians have experienced with their patients during the shared decision-making process. These have some implications for the design of a decision aid.

\subsection{Delivery: Recognize me as a unique individual, part of a group}

4.3.1 Patients. In the process of getting evaluative feedback regarding the design of the existing decision aid, our focus group participants expressed a desire to be treated with empathy, as unique individuals with different lived experiences.

During the focus groups, one topic that sparked a lot of discussion was how health resources and professionals treated smokers. As smoking is recognized as a major risk factor of lung cancer, many health education materials and healthcare professionals emphasize the need to quit smoking. Smokers know that they should quit, but they felt people should acknowledge how they came to be smokers (e.g., it was the norm), and stayed being smokers (e.g., as a result of different life stressors). As we heard in a focus group:

"It's long time ago. Smoking was just..., they did not tell us it is harmful. You turn on TV and see all these glamorous movie stars, they smoke and so you did not really think that smoking was bad back in the day... and when you were young, it was cool that you could hold cigarettes..." - Female smoker, focus group (African American)

Another demonstration of why this recognition is important is how smoking habits are asked in the decision aid. For instance, it features a risk calculator that takes a person's demographic information and smoking history as input and calculates the risk of getting lung cancer. One of the problems raised by participants is that the calculator assumes a person can accurately recall and calculate their smoking history, when one often smokes more (or less) or quit due to changing life circumstances.

While expressing the need to be treated as an individual with a unique relationship with smoking, we noticed that participants working through the decision aid in pairs stimulated lively discussions about the materials. This observation suggests that the decision-making process could be enhanced with a peer or group (or simply social) learning environment. With people who share and understand similar lived experiences (e.g., smokers who have been smoking since their teens), people found that they were able to "find their voice" and have a conversation about the decision they were considering in a safe and non-judgmental context. During and after focus groups, the facilitator received multiple instances of feedback reflecting the benefits of learning and discussing the decision in a peer or group setting, as opposed to patient-doctor pairing where they may feel judged for being a smoker.

\section{DISCUSSION}

Through participatory design workshops and focus groups, and triangulating these findings with two physicians experienced with lung cancer screening, we identified three themes - vocabulary, time, and delivery. Below, we present design suggestions for customizing a web-based decision aid for a relatively new medical procedure, lung cancer screening. These suggestions are developed with the goal of providing decision aid designers with some tangible tools to complement the existing content-oriented guidelines [18].

\subsection{Bridging the Gap: Decision Aid as a Mediator for Patient and Physician}

Our findings suggested that a decision aid of an unknown medical procedure (i.e., lung cancer screening) for traditionally underscreened populations needs to have two functions: raising awareness, and facilitating the transition from awareness to action (i.e., consult with physicians). To do so, one crucial step is for decision aid designers to make a concerted effort to bridge the vocabulary and language used by patients and physicians.

5.1.1 Raising Awareness through Relevance. Conveying a sense of relevance is key to raising awareness through an online decision aid. Decision aid developers could make the tool more approachable by presenting information through perspectives people can relate with. To provide an entry point without swaying people from the outset, which was a concern for the physicians, we recommend showing relatable perspectives from those who decided to get screened, as well as those who decided not to. The perspectives shown can focus on the thought process and experience working with different stakeholders before reaching the decision, and reflection after the decision (or screening). We believe this is pertinent when there is uncertainty about people's attitudes and knowledge with regards to the target medical procedure, as it could increase the chances of visitors having a positive first impression and staying engaged [39] in other important content the decision aid intends to promote.

Existing work on how patients manage chronic conditions has shown that patients and caregivers find connecting with people with similar experiences (i.e., as patients or as caregivers) to be helpful, as they provide the necessary emotional support in addition to purely informational support $[41,45]$. They also have the capability to translate general health information that is sensitive to contextual factors such as socioeconomic background and living environment [19]. The feeling that "this person understands me" is really important, as workshop participants have repeatedly emphasized the importance of shared experience for the design of the landing page. For instance, participants designed a decision aid with a landing page featuring a person with a similar background explaining screening, as shown in Figure 2. We would like to stress that this general principle should be applied throughout the decision aid and not just the landing page, as it is common for people to reach an arbitrary page on a site through search engines for health information [10].

Here is an example of implementing this design suggestion to provide relatable perspectives to highlight the relevance of the decision in question to their target audience. To tailor information presented to users, a decision aid could present people's perspectives in the form of questions that people with different profiles (e.g., history of smoking, age, sex/gender) frequently ask, and connect users to suggested relevant resources. For instance, "I have been smoking for X years, should I ...?", "I used to smoke, but I have stopped. Am I ...?" The questions could also be presented in the view 


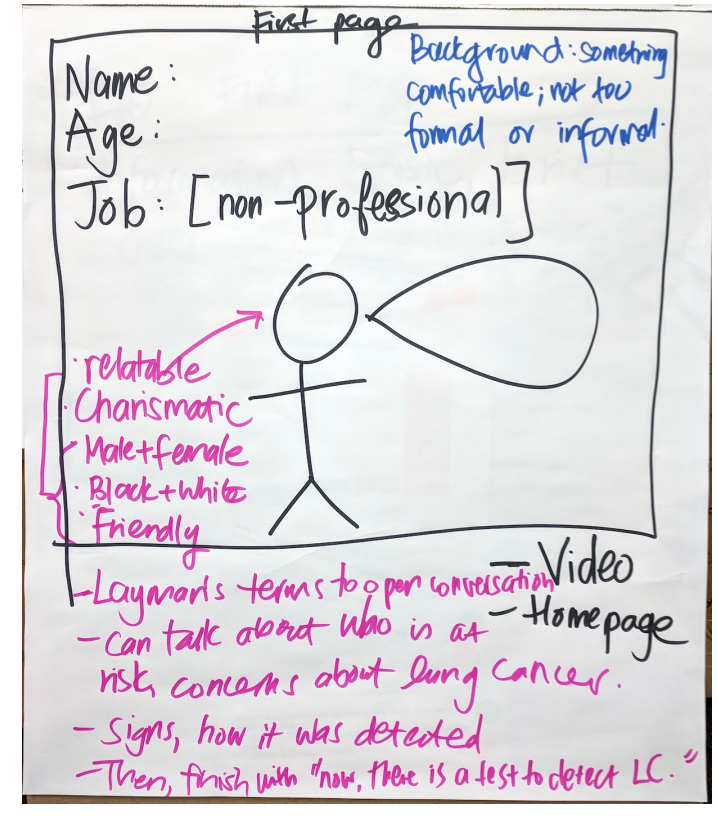

Figure 2: Landing page designed by a Participatory Design Group (African American) with a relatable person

of health professionals to help overcome people's (i.e., smokers) fear of cancer and stigma to initiate learning, thinking, and action (e.g., visiting a doctor) [27]. The provided responses could be further supplemented by stories - through text, storyboards, or videos [24] - from patients, caregivers, and clinicians that detail their own experience before, during, or after screening to help individuals see the perspectives of someone who had a similar experience (or a clinician's observation).

5.1.2 Connecting Everyday Language and Medical Vocabulary. Designing an online decision aid that appeals to traditionally underscreened population is challenging as it can easily lose the audience if the content does not "speak to them." With the general public having relatively low levels of literacy, health literacy [35], and graph/numerical literacy [38], it is not surprising that we found our participants had trouble with the information presented as well. Patients unfamiliar with medical vocabulary might stop or limit their engagement with the decision aid, or worse, prevent them from working with health professionals [30]. This unfamiliarity is likely to disproportionately affect people with low socioeconomic status and lower levels of literacy, and may exacerbate existing disparities of the lung cancer burden. Joseph-Williams et al. [18] have suggested field-testing and controlling the reading level, but they do not give specific directions for improvement. On the other hand, if potential patients do consider taking action and consult with physicians, decision aids should equip patients with the vocabulary and knowledge to empower them to be active participants in the shared decision-making process. Using layman's terms, while desirable, is not enough: decision aids need to bridge the gap between potential patients' and medical professionals' languages so that they can better interact with each other during the shared decision-making process.
Existing literature suggests various ways to tackle the issue of literacy using multimedia [20,49], crowdsourcing translation of existing materials $[6,23]$, providing definition and analogy $[31,38,38]$, and virtual agents to provide structured information [2,25], with the central theme of using the more accessible layman's terms, particularly for people with low levels of literacy. Building on existing literature, we propose to use everyday language, not as a replacement, but potentially to augment existing medical discourse/thinking to facilitate understanding. This will allow both medical professionals and people without medical training (e.g., patients and caregivers) to not only understand the concepts, but prepare them for the discussion. This is likely to be beneficial, as illiteracy has been shown to cause shame and prevent people from approaching clinicians [30], while clinicians also need to acquire skills to communicate with patients effectively [48].

Here, we describe an example of applying this suggestion that can be used to improve an existing decision aid. A hover-over dictionary can be developed to enhance an existing web-based decision aid to provide layman annotations for formal medical descriptions, and vice versa. For instance, to explain the benefits of screening (e.g., lung cancer mortality risk reduction), an entry could be created to elaborate on the tangible benefits that people can easily understand, such as living longer and spending more time with family. The key feature of this dictionary is to allow stakeholders to contribute their ways of describing a medical condition or procedure. This feature allows not only patients, but also clinicians to collaboratively provide alternatives for explaining different medical discourse. From our interviews with physicians, we found that their engagement with patients requires them to find effective ways to explain different concepts to patients. Allowing stakeholders to collaboratively curate such a dictionary can facilitate the sharing of these beneficial patient-doctor communication approaches.

5.1.3 Foster Shared Understanding with Concrete Take-Home Message. In addition to managing the discrepancy between patients and physicians' vocabulary, we have also identified a critical issue that might render a decision aid ineffective: patients do not have the necessary training to interpret scientific facts as medical professionals do. Decision aid developers should take care not to let succinctly presented, scientifically accurate information overshadow the delivery of a clear message and explanation of the rationale to patients. Otherwise, confusion and stress about understanding the main message might drain a patient's energy and affect the efficacy of the decision aid.

One plausible solution to tackle the dilemma between being concise and provide information (e.g., explanations) that might overwhelm users [4] is to apply the idea of training wheels for user interface design proposed by Carrol and Carrithers [5]. Decision aid developers could consider getting the core messages out by directing visitors' attention to those messages and progressively reveal more information/explanations as needed. This will likely reduce the barrier for our population with low literacy and computer skills. For instance, one can add a topic message or explanation card deck that will show visitors the major message, takeaway, or explanation to different locations where information is presented (e.g., in text or charts). The deck can be customized to appear before or after 
users have engaged with the materials, to progressively reveal or reemphasize information.

\subsection{Bending Time: Decision Aid as a Time Machine to Navigate the Screening Process}

Through triangulating feedback from patients and physicians, we learnt that using a timeline to structure the information presented in a decision aid not only could be a viable way to help patients navigate through health information in a structural manner, but also has the potential to help patients understand that lung cancer screening is a process, instead of a one-time event. To accommodate differences between laypersons and health professionals' views on the target medical procedure as a whole, decision aid developers could consider providing different overlays (e.g., menu, annotation, or navigation aid) that highlight the process-oriented views of different stakeholders. Providing organization of materials based on the sequence of events will likely help people see the big picture, and access content on the decision aid in a structure that aligns with their thinking about a medical procedure, without being overwhelmed by information.

As we have found that patients have different conceptualization of time, possibly due to their current interest (e.g., understanding, considering, under-going lung cancer screening), decision aid developers could further consider allowing navigation facilities (e.g., menu) to selectively "zoom-in" to unfold information relevant to a specific time period while hiding information more relevant to other stages. For instance, the decision aid could allow users to zoom in to the "understanding" phase where background and main idea of lung cancer screening is introduced, while entries for actual screening procedure and follow-up steps will be aggregated and revealed only if users decide to understand more. On the other hand, users can also choose to "zoom-out" by folding the details of the actual screening procedure and digest information in a "before-after" mindset.

Here is an example of how to make use of the design suggestion to guide visitors. As stakeholders might have different views of important events [4,31], a decision aid can support customizable roadmaps that can be tailored by different groups of audience [6] to intuitively guide users across different materials. For example, the decision aid can have a layperson mode or physician mode [31] of presenting information, depending on who is navigating the decision aid. We argue that, while having different ways of accessing could be confusing and challenging for people with a low level of computer literacy [27], allowing different roadmaps displayed simultaneously is a good opportunity for decision aids to help stakeholders understand everyone's perspectives. In fact, the ability to bridge the gap between different stakeholders' perspectives could potentially help patients (and physicians) prepare for a productive clinical encounter.

\subsection{Creating Space: Decision Aid with Inclusive Decision Support}

This design suggestion aims at accommodating people's need to be recognized as individuals, each with their own reasons for starting, continuing, and quitting smoking. In our study, lung cancer screening appeals to current smokers and former smokers very differently, with current smokers often preferring not to know the status of their lungs. To appeal to both groups, we should be cognizant of the framing of the message. Indeed, former smokers in our focus groups were very keen on using "scare tactics" to get current smokers to quit and get their lungs checked. Unsurprisingly, this was not what the current smokers wanted to see. Relatedly, this leads us to the observation that former-smoker groups and current-smoker groups have high within-group rapport, where the stigma of being a smoker becomes less of an issue, suggesting that decision aid developers should consider providing opportunities to facilitate peer or group activities. By inviting similar and yet different individuals to support each other in the decision-making process, stigma may play a lesser role and users could engage with the decision aid with an open mind. As online peer interaction has been suggested to be important for understanding medical information and making decisions [34], decision aid developers should actively think about different ways of nurturing such environments through digital tools or in-person activities. Here are two examples of adding a social layer to a decision aid to improve user experience.

Learn together with social traces: A decision aid can collect data detailing visitors' behaviors, or traces, and present them in different forms to other visitors to create an online social learning experience. Moreover, a decision aid can provide input mechanisms and invite users to contribute while they are using the site. For instance, one way for users to organize their learning is to allow highlighting and annotations, so that users can curate content that they find useful or need further clarification (e.g., with doctors). With the annotations, the decision aid can add a social layer on top of the content, displaying commentary such as " 465 people have also saved this section for review later." The social layer allows users to engage with the decision aid with asynchronous participation from other users.

Community event drop-in: An opportunity to create peer/group learning environments is to actively participate in community events to introduce the decision aid to local communities. Given that a significant portion of our target population may have low literacy and/or computer skills, publicizing the decision aid to raise awareness among this population is necessary. In fact, one question that was brought up by our participants in our focus groups was "how do people actually find this thing [the online decision aid]?" Our participants and the community partners we worked with suggested that we should introduce the tool at community events such as health fairs or advertise in the local newspapers to broaden our reach.

\subsection{Designing Decision Aids: Using Lung Cancer as an Example}

By situating the design of an existing patient-centered decision aid where patients would access it themselves without a health professional being present, we discovered several challenges for users and uncovered some discrepancies in beliefs held by patients and physicians that warrant attention of decision aid designers. We have attempted to mitigate some of the discrepancies in our design suggestions, in the hopes that the decision aid could raise 
the awareness of such conflicts and create opportunities for stakeholders (e.g., patients, physicians, and decision aid designers) to collaboratively improve the inclusivity of web-based decision aids.

While the above suggestions are presented separately, they can also be combined to generate new design ideas that could potentially enhance existing decision aids to support individuals outside the context of a doctor's office. For instance, combining event-based information flow with everyday language might suggest showing navigation using traditional medical terminology and everyday language in parallel. Patients can then access content in an eventbased structure written in everyday language, but also allow them to see the differences in terminology and conceptualization between themselves and the health professionals with whom they work.

When we place decision aid design in a pervasive environment, our findings also offer important lessons to personal informatics and health applications in the future. In this scenario, more biomedical, behavioral and emotional data could be readily collected and analyzed to assist in the decision-making process. Here, a decision aid could continuously monitor a patient's health data, so as to engage the patient with relevant resources throughout the healthseeking journey to support different types of decision-making. For example, if test results indicated that more tests were required, the nature of these additional tests could be explained. Or, if an individual's smoking behavior were tracked, we could recommend various cessation resources at different points in time based on the data collected. However, in this scenario, there are no healthcare professionals available to patients for immediate consultation. As patient sensitive data are involved, it will be ethically important for a decision aid in a pervasive environment to provide adequate support and resources to guide patients to understand what data would be collected and for what purposes. Certainly, there may be concerns about privacy given the continuous monitoring, along with insurance-sensitive health issues.

Moreover, in light of the discrepancies between the medical care patients themselves perceive they should get and what is deemed clinically beneficial, our findings suggest that any results and analysis delivered to patients will have to be done carefully with the appropriate language. A potential solution could be a system that visualizes how different kinds of data are needed at different waypoints in a patient's health journey. Specifically, patients and healthcare providers can use a system with software critics [8] that provide timely explanations and identify possible misunderstandings generated from the discrepancies described above. This kind of system has the potential to improve mutual understanding and help negotiate the ways in which to implement data sharing realistically to support shared decision-making.

We hope that the suggestions and examples presented above offer decision aid developers a set of tools to improve the user experience of decision aids, with the particular goal of creating inclusive decision aids for people with low literacy. These suggestions are by no means exhaustive, and field testing the suggested features is still needed. Moreover, the small number of patients from African American and Latinx populations might prevent us from seeing nuanced differences between these two communities. The uneven number of stakeholders of each kind could also lead to an unbalanced representation of viewpoints. Nonetheless, the design suggestions are generated based on co-designing and evaluating with potential users, and in deliberation with physicians and decision aid developers. We believe that our suggestions can serve as additional guidelines to complement the existing patient decision aid guidelines that are primarily content-oriented $[16,18]$.

\section{CONCLUSION AND FUTURE WORK}

In this project, we attempted to tackle the critical issue of underscreening of lung cancer through the design of an online lung cancer screening decision aid. We employed PD to engage with African American and Latinx populations who typically underscreen for cancer to inform an inclusive design for older adults with lower levels of health and general literacy. The co-design activity revealed several challenges and hinted at potential solutions. Based on our analysis, we identified three themes: vocabulary, time, and delivery, with a set of design suggestions to complement existing content-oriented guidelines $[7,18]$ that are well-established within the medical and public health communities. Future work includes incorporating additional stakeholders' (e.g., primary care physicians) input into the design suggestions, re-engaging study participants to validate our findings and suggestions, and refining design suggestions through working with decision aid developers to evaluate the effectiveness of applying these suggestions through the iterative design and evaluation process.

\section{ACKNOWLEDGMENTS}

We would like to thank: our community partners Eastside Community Network and Community Health and Social Services (CHASS) Center for their tremendous support; Dr. Kirsten Herold for editing earlier drafts; our Spanish group facilitators Jean Carlos Rodrigo Diaz and Evelyn Jimenez Mendoza for their assistance; past and present members of the ShouldIScreen research team for their input; and lastly, our study participants for their invaluable insight. This work was funded by the National Cancer Institute under Award No P30CA046592 and the University of Michigan Rogel Cancer Center Cancer Control and Population Sciences Research Program: Outreach and Health Disparities Grant. The content is solely the responsibility of the authors and does not necessarily represent the official views of the National Institutes of Health.

\section{REFERENCES}

[1] P.B. Bach, M.W. Kattan, M.D. Thornquist, M.G. Kris, R.C. Tate, M.J. Barnett, L.J. Hsieh, and C.B. Begg. 2003. Variations in lung cancer risk among smokers. Journal of the National Cancer Institute 95, 6 (2003), 470-478.

[2] Timothy W. Bickmore, Laura M. Pfeifer, and Brian W. Jack. 2009. Taking the Time to Care: Empowering Low Health Literacy Hospital Patients with Virtual Nurse Agents. In Proceedings of the SIGCHI Conference on Human Factors in Computing Systems (CHI '09). ACM, New York, NY, USA, 1265-1274.

[3] Alison T Brenner, Teri L Malo, Marjorie Margolis, Jennifer Elston Lafata, Shynah James, Maihan B Vu, and Daniel S Reuland. 2018. Evaluating shared decision making for lung cancer screening. JAMA Internal Medicine (2018).

[4] Ayşe G. Büyüktür and Mark S. Ackerman. 2017. Information Work in Bone Marrow Transplant: Reducing Misalignment of Perspectives. In Proceedings of the 2017 ACM Conference on Computer Supported Cooperative Work and Social Computing (CSCW'17). ACM, New York, NY, USA, 1740-1752.

[5] John M. Carroll and Caroline Carrithers. 1984. Training Wheels in a User Interface. Commun. ACM 27, 8 (Aug. 1984), 800-806.

[6] Tao Dong, Mark S. Ackerman, Mark W. Newman, and Gaurav Paruthi. 2013. Social Overlays: Collectively Making Websites More Usable. In Human-Computer Interaction - INTERACT 2013 (Lecture Notes in Computer Science), Paula Kotzé, Gary Marsden, Gitte Lindgaard, Janet Wesson, and Marco Winckler (Eds.). Springer Berlin Heidelberg, 280-297. 
[7] Glyn Elwyn, Annette O'Connor, Dawn Stacey, Robert Volk, Adrian Edwards, Angela Coulter, Richard Thomson, Alexandra Barratt, Michael Barry, Steven Bernstein, et al. 2006. Developing a quality criteria framework for patient decision aids: online international Delphi consensus process. BM7 333, 7565 (2006), 417.

[8] Gerhard Fischer, Andreas C. Lemke, Thomas Mastaglio, and Anders I. Morch 1990. Using Critics to Empower Users. In Proceedings of the SIGCHI Conference on Human Factors in Computing Systems (CHI '90). ACM, New York, NY, USA, 337-347.

[9] Agency for Healthcare Research and Quality. 2016. Is Lung Cancer Screening Right For Me? https://effectivehealthcare.ahrq.gov/decision-aids/ lung-cancer-screening/patient.html [Online; accessed 14 Aug 2018].

[10] Susannah Fox and Maeve Duggan. 2013. Health Online 2013. http://www pewinternet.org/2013/01/15/health-online-2013/ [Online; accessed 14 Aug 2018]

[11] Michael Gonzales and Laurel Riek. 2013. Co-designing Patient-centered Health Communication Tools for Cancer Care. IEEE. https://doi.org/10.4108/icst pervasivehealth.2013.252109

[12] National Cancer Institute. 2017. Health Information National Trends Survey (HINTS) 5, Cycle 1. https://hints.cancer.gov [Dataset online; accessed 15 August 2018].

[13] Maia L. Jacobs, James Clawson, and Elizabeth D. Mynatt. 2014. My Journey Compass: A Preliminary Investigation of a Mobile Tool for Cancer Patients. In Proceedings of the SIGCHI Conference on Human Factors in Computing Systems (CHI '14). ACM, New York, NY, USA, 663-672. https://doi.org/10.1145/2556288.2557194

[14] Nancy K Janz and Marshall H Becker. 1984. The Health Belief Model: A decade later. Health Education Quarterly 11, 1 (1984), 1-47.

[15] Ahmedin Jemal and Stacey A Fedewa. 2017. Lung cancer screening with low-dose computed tomography in the United StatesâĂT2010 to 2015. JAMA Oncology 3, 9 (2017), 1278-1281.

[16] Tamara S Jensen, Joseph Chin, Lori Ashby, Jamie Hermansen, and Joseph D Hutter. 2015. Decision Memo for Screening for Lung Cancer with Low Dose Computed Tomography (LDCT) (CAG-00439N). https://www.cms.gov/ medicare-coverage-database/details/nca-decision-memo.aspx?NCAId=274 [Online; accessed 10. Sep. 2018]

[17] Masahito Jimbo, Gurpreet K Rana, Sarah Hawley, Margaret Holmes-Rovner Karen Kelly-Blake, Donald E Nease Jr, and Mack T Ruffin IV. 2013. What is lacking in current decision aids on cancer screening? CA: A Cancer fournal for Clinicians 63, 3 (2013), 193-214.

[18] Natalie Joseph-Williams, Robert Newcombe, Mary Politi, Marie-Anne Durand, Stephanie Sivell, Dawn Stacey, Annette O'Connor, Robert J. Volk, Adrian Edwards, Carol Bennett, Michael Pignone, Richard Thomson, and Glyn Elwyn. 2014 Toward Minimum Standards for Certifying Patient Decision Aids: A Modified Delphi Consensus Process. Medical Decision Making 34, 6 (Aug. 2014), 699-710.

[19] Elizabeth Kaziunas, Mark S Ackerman, and Tiffany CE Veinot. 2013. Localizing Chronic Disease Management: Information Work and Health Translations. In American Society for Information Science \& Technology (ASIS\&T) Annual Conference.

[20] Elizabeth Kaziunas, Pei-Yao Hung, and Mark S. Ackerman. 2014. FIT2: Information Translations for Health Practices. In Proceedings of The 2nd International Workshop on Collaboration and Coordination in the Context of Informal Care (CC$\mathrm{CiC}$ ) in ACM Conference on Supporting Groupwork (CCCiC in GROUP '14). ACM, Sanibel Island, USA.

[21] Yan Kwan Lau, Tanner J. Caverly, Pianpian Cao, Sarah T. Cherng, Mindy West Charles Gaber, Douglas Arenberg, and Rafael Meza. 2015. Evaluation of a Person alized, Web-Based Decision Aid for Lung Cancer Screening. American fournal of Preventive Medicine 49, 6 (Dec. 2015), e125-e129.

[22] Yan Kwan Lau, Tanner J Caverly, Sarah T Cherng, Pianpian Cao, Mindy West Douglas Arenberg, and Rafael Meza. 2014. Development and Validation of a Personalized, Web-Based Decision Aid for Lung Cancer Screening Using Mixed Methods: A Study Protocol. FMIR Research Protocols 3, 4 (Dec. 2014).

[23] Adrian Laurenzi, Megumu Brownstein, Anne M. Turner, Julie A. Kientz, and Katrin Kirchhoff. 2013. A Web-based Collaborative Translation Management System for Public Health Workers. In CHI '13 Extended Abstracts on Human Factors in Computing Systems (CHI EA '13). ACM, New York, NY, USA, 511-516.

[24] Leslie S. Liu, Jina Huh, Tina Neogi, Kori Inkpen, and Wanda Pratt. 2013. Health Vlogger-viewer Interaction in Chronic Illness Management. In Proceedings of the SIGCHI Conference on Human Factors in Computing Systems (CHI '13). ACM, New York, NY, USA, 49-58.

[25] Sanjana Mendu, Mehdi Boukhechba, Janna R. Gordon, Debajyoti Datta, Edwin Molina, Gloria Arroyo, Sara K. Proctor, Kristen J. Wells, and Laura E. Barnes. 2018 Design of a Culturally-Informed Virtual Human for Educating Hispanic Women About Cervical Cancer. In Proceedings of the 12th EAI International Conference on Pervasive Computing Technologies for Healthcare (PervasiveHealth '18). ACM, New York, NY, USA, 360-366.

[26] Virginia A Moyer. 2014. Screening for lung cancer: US Preventive Services Task Force recommendation statement. Annals of Internal Medicine 160, 5 (2014), $330-338$
[27] Julia Mueller, Alan Davies, Simon Harper, Caroline Jay, and Chris Todd. 2016. Widening Access to Online Health Education for Lung Cancer: A Feasibility Study. In Proceedings of the 13th Web for All Conference (W4A '16). ACM, New York, NY, USA, 35:1-35:4.

[28] Krapcho M Miller D Brest A Yu M Ruhl J Tatalovich Z Mariotto A Lewis DR Chen HS Feuer EJ Cronin KA (eds) Noone AM, Howlader N. 2018. SEER Cancer Statistics Review, 1975-2015. https://seer.cancer.gov/csr/1975_2015/ National Cancer Institute. Bethesda, MD. [Online; accessed 1 Sep 2018].

[29] A.M. O'Connor, D. Stacey, and M.J. Jacobsen. 2011. Ottawa decision support tutorial: improving practioners' decision support skills. Ottawa Hospital Research Institute, Ottawa. https://decisionaid.ohri.ca/ODST/pdfs/ODST.pdf

[30] Nina S. Parikh, Ruth M. Parker, Joanne R. Nurss, David W. Baker, and Mark V. Williams. 1996. Shame and health literacy: the unspoken connection. Patient Education and Counseling 27, 1 (Jan. 1996), 33-39.

[31] Sun Young Park, Yunan Chen, and Shriti Raj. 2017. Beyond Health Literacy: Supporting Patient-Provider Communication During an Emergency Visit. In Proceedings of the 2017 ACM Conference on Computer Supported Cooperative Work and Social Computing (CSCW' 17). ACM, New York, NY, USA, 2179-2192.

[32] James O Prochaska and Wayne F Velicer. 1997. The transtheoretical model of health behavior change. American fournal of Health Promotion 12, 1 (1997), 38-48.

[33] Daniel S Reuland, Laura Cubillos, Alison T Brenner, Russell P Harris, Bailey Minish, and Michael P Pignone. 2018. A pre-post study testing a lung cancer screening decision aid in primary care. BMC Medical Informatics and Decision Making 18, 1 (2018), 5.

[34] Ellen L. Rubenstein. 2012. "Things my doctor never told me": Bridging information gaps in an online community. Proceedings of the American Society for Information Science and Technology 49, 1 (Jan. 2012), 1-10.

[35] Rima Rudd, Irwin Kirsch, and Kentaro Yamamoto. 2004. Literacy and Health in America. Policy Information Report. Educational Testing Service (2004).

[36] Douglas Schuler and Aki Namioka. 1993. Participatory Design: Principles and Practices. CRC Press. Google-Books-ID: pWOEk6Sk4YkC.

[37] SEER. 2018. SEER Cancer Statistics Factsheets: Common Cancer Sites. https:// seer.cancer.gov/statfacts/html/common.html National Cancer Institute. Bethesda, MD. [Online; accessed 14 Aug 2018].

[38] Joseph Sharit, Miriam Lisigurski, Allen D. Andrade, Chandana Karanam, Kim M. Nazi, James R. Lewis, and Jorge G. Ruiz. 2014. The Roles of Health Literacy, Numeracy, and Graph Literacy on the Usability of the VA's Personal Health Record by Veterans. F. Usability Studies 9, 4 (Aug. 2014), 173-193.

[39] Elizabeth Sillence, Pam Briggs, Peter Harris, and Lesley Fishwick. 2007. Health Websites that people can trust - the case of hypertension. Interacting with Computers 19, 1 (Jan. 2007), 32-42.

[40] Gerard A Silvestri, Paul J Nietert, James Zoller, Cindy Carter, and David Bradford. 2007. Attitudes towards screening for lung cancer among smokers and their non-smoking counterparts. Thorax 62, 2 (2007), 126-130.

[41] Meredith M. Skeels, Kenton T. Unruh, Christopher Powell, and Wanda Pratt. 2010. Catalyzing Social Support for Breast Cancer Patients. In Proceedings of the SIGCHI Conference on Human Factors in Computing Systems (CHI '10). ACM, New York, NY, USA, 173-182. https://doi.org/10.1145/1753326.1753353

[42] Jennifer Stuber, Sandro Galea, and Bruce G Link. 2008. Smoking and the emergence of a stigmatized social status. Social Science \& Medicine 67, 3 (2008), $420-430$.

[43] Judith Swan, Nancy Breen, Barry I Graubard, Timothy S McNeel, Donald Blackman, Florence K Tangka, and Rachel Ballard-Barbash. 2010. Data and trends in cancer screening in the United States: results from the 2005 National Health Interview Survey. Cancer 116, 20 (2010), 4872-4881.

[44] National Lung Screening Trial Research Team. 2013. Results of initial lowdose computed tomographic screening for lung cancer. New England fournal of Medicine 368, 21 (2013), 1980-1991.

[45] Hilda Tellioğlu, Myriam Lewkowicz, Aparecido Fabiano Pinatti De Carvalho, Ivan Brešković, and Marén Schorch. 2014. Collaboration and Coordination in the Context of Informal Care (CCCiC 2014). In Proceedings of the Companion Publication of the 17th ACM Conference on Computer Supported Cooperative Work \& Social Computing (CSCW Companion '14). ACM, New York, NY, USA, 339-342. https://doi.org/10.1145/2556420.2558862

[46] Kristin Voigt. 2010. Smoking and social justice. Public Health Ethics 3, 2 (2010), 91-106.

[47] Elizabeth Ward, Ahmedin Jemal, Vilma Cokkinides, Gopal K Singh, Cheryll Cardinez, Asma Ghafoor, and Michael Thun. 2008. Cancer disparities by race/ethnicity and socioeconomic status. CA: A Cancer fournal for Clinicians 54, 2 (2008), 78-93.

[48] Mark V Williams, Terry Davis, Ruth M Parker, Barry D Weiss, et al. 2002. The role of health literacy in patient-physician communication. Family Medicine 34, 5 (2002), 383-389.

[49] Bo Xie, Tom Yeh, Greg Walsh, Ivan Watkins, and Man Huang. 2012. Co-designing an e-Health Tutorial for Older Adults. In Proceedings of the 2012 iConference (iConference '12). ACM, New York, NY, USA, 240-247. 